\title{
Energy Efficient Heuristic Base Job Scheduling Algorithms in Cloud Computing
}

\author{
Jaspreet Singh, Deepali Gupta \\ ${ }^{I}$ (Ph.D Research Scholar, Dept of CSE, MM University, Sadopur, Ambala, India) \\ ${ }^{2}$ (Professor \& Head, Dept of CSE, MM University, Sadopur, Ambala, India)
}

\begin{abstract}
Cloud computing environment provides the cost efficient solution to customers by the resource provisioning and flexible customized configuration. The interest of cloud computing is growing around the globe at very fast pace because it provides scalable virtualized infrastructure by mean of which extensive computing capabilities can be used by the cloud clients to execute their submitted jobs. It becomes challenge for the cloud infrastructure to manage and schedule these jobs originated by different cloud users to available resources in such a manner to strengthen the overall performance of the system. As the number of user increases the job scheduling become an intensive task. Energy efficient job scheduling is one constructive solution to streamline the resource utilization as well as to reduce the energy consumption. Though there are several scheduling algorithms available, this paper intends to present job scheduling based on two Heuristic approaches i.e. Efficient MQS (Multi-queue job scheduling) and ACO (Ant colony optimization) and further evaluating the effectiveness of both approaches by considering the parameter of energy consumption and time in cloud computing.
\end{abstract}

Keywords: Cloud Computing, Energy Efficient Job Scheduling, Efficient MQS in Cloud Computing, ACO

\section{Introduction}

The name cloud computing was came from the cloud symbol that is most commonly used to portray the Internet in flow charts and diagrams. Cloud Service providers and cloud end-users are the key players for the cloud computing paradigm. Based upon the dynamic requirements the cloud infrastructure focused to provide the computing environment to end user with QOS (Quality of Service) and make profits for the cloud service providers. Cloud environment charging pay per use from the cloud clients over the internet and is entitled by virtualization and the abstraction technology. So this environment is a distributed paradigm spread over the large scale which is providing the on demand services such as non interfering resource pooling, rapid elasticity and location independence with the help of virtualization support and hides the details of system implementation from cloud users with abstraction support [1] [16]. The paper is organized as follows: In Section II the discussion done about the architecture, characteristics of cloud computing followed by suitable example of cloud environment. In section III, we have discussed about job scheduling techniques in cloud computing. In section IV discussion is made on heuristic base job scheduling techniques i.e. Efficient Multi-queue job scheduling (MQS) and Ant colony optimization (ACO) in Cloud computing environment. Section V discuss about comprehensive experimental results achieved by implementing from both the technique. Conclusion and future work can be found in the section VI.

\section{Cloud Computing Architecture}

The model of cloud offers services that can be grouped in three classes mentioned below:

- Software as a Service: SaaS provide the service to end user over the internet to develop application as demanded by the user. Zero maintenance cost is required at the user side because by the mean of internet, users are able to use the software and the service provider is responsible for managing the software [1] [16].

- Platform as a Service: All resources needed to build an application is provided by PaaS. It provides support of operating system and software development framework on which user can build and host their own applications [1].

- Infrastructure as a Service: IaaS allows clients with the access to server hardware, storage and bandwidth. The clients of IaaS pay on per user basis like by the hour, week or month [1].

(A) The Essential Types and Characteristics of Cloud Computing:

The environment of cloud computing can be viewed in three parts Public Cloud, Private Cloud and Hybrid Cloud [8]. A Public Cloud is made available in a pay-as-you-go manner and is owned by a third-party cloud provider. A private cloud makes services accessible for one designated organization and is restricted to employees and trusted partners of that organization. Hybrid clouds are combinations of public and private clouds that work together. In this model, outsourcing of processing and non critical information is done to public cloud while the data and services which are significant to the business are kept in control [11]. Some of the 
essential characteristics of cloud computing includes On-demand Service, Resource Pooling, Reliability, Scalability and Rapid Elasticity [2].

(B) Some Examples Associated with Cloud Environment are described below :

- Amazon's Elastic Computing Cloud (EC2) Provides resizable compute capacity (CPU cycles) to users. Amazon EC2 decreases the time required to obtain and boot new virtual server instances to minutes, and allow us to configure security [11].

- Amazon's Simple Storage Service (S3) provide provides the web service interface by virtue of which we can store and retrieve any amount of data at any instant from anywhere by internet [11].

- CRM services provided by salesforce.com which can manage customer information like name, address, phone no etc and keep track on the customer activity [11].

\section{Job Scheduling Techniques in Cloud Computing}

Job Scheduling is the process of deciding the distribution of the virtual resources between the several different user jobs. The objective of job scheduling is to achieve high system throughput and minimizing total processing time. Based upon the certain parameter the job scheduler allocates the different user jobs to available resources. Each resource processes the assigned job and the result is returned by the resources to scheduler. Max throughput, Min turnaround time, Min waiting time, Min response time and High CPU Utilization are the several scheduling criteria that scheduler should consider when scheduling user job to adaptable resources in a adaptable time. So cloud job scheduling is the NP complete problem. The solution to this problem is given by the Enumeration method, Heuristic method and by Approximation method. Enumeration Method, selects an optimal solution if all possible solution are enumerated and compared one by one, but when no of instances is large exhaustive enumeration is not feasible then in that case heuristic uses suboptimal algorithm to find good solution in a fast manner. Approximate Algorithms finds the approximate solution to optimized solution [3] [8] [10]. The process of scheduling in cloud computing can be concluded in three stages as mentioned below [3]:

- Resource Discovery and Filtering: Searching of resources is done in the network and their related status information is collected out.

- Resource Selection: Based upon the certain parameter the target resource is selected.

- Task Submission: User task is submitted to the selected resource for processing.

Based upon the work done in [9] classification of task scheduling is done as in the distributed environment the scheduling method composes of Heuristic and Hybrid Technique. Heuristic method performs the Static and Dynamic Scheduling. Static Scheduling Method works on the principle that user job arrive at the same period of time and they are independent of the system resource's states and their availability. In Dynamic Scheduling Method jobs arrive at different period of time and it is dependent on the system machine state. The various fundamental components for cloud computing scenario are described below:

- Computing entity: All the Virtual Machines providing the computing facilities like operating system, software etc. is present in the cloud system environment to execute the user submitted jobs [9].

- Job scheduler: It is responsible to decide execution order of the user jobs by selecting the optimal scheduling strategy [9].

- Job waiting queue: It holds number of jobs which are waiting to get assigned on a particular machine to get executed [9].

(A) Existing Job Scheduling Algorithms

The several types of scheduling algorithms are First Come First Serve, Shortest Job First, Round

Robin, Priority Scheduling, Max-Min and Min-Min Scheduling while some meta-heuristic algorithm include ACO(Ant Colony Algorithm), GA(Genetic Algorithm) etc, all are briefly defined as follow [12] :

1. First Come First Serve (FCFS) scheduling, the jobs are queued in the order in which they arrived and jobs are assigned to the resources once they become available [3].

2. Shortest Job First Scheduling Algorithm (SJF) picks the job with the least execution time. Highest Priority is assigned to the jobs with minimum execution time and placed first in queue while the lowest priority is assigned to the jobs with the maximum execution time placed at end of queue [3].

3. Round Robin Scheduling Algorithm (RR) is based on the principal of time quantum. Several jobs are dispatched in FCFS logic and the time slice associated with the process decides the duration of allocation [14].

4. Priority scheduling algorithm based upon the straightforward process that priority is assigned to each job and priority in turn define the execution order of the several job in the queue [3].

5. In Min-Min scheduling algorithm, firstly the execution of smallest job is carried out and larger job have to wait for more time [3] [14].

6. Max-Min scheduling algorithm follow the reverse process of Min-Min Scheduling algorithm, the criteria of this algorithm based upon executing the largest job(with maximum completion time) first on best machines later the small jobs are executed which further takes long time [3] [14]. 


\section{Heuristic Base (MQS And ACO) Job Scheduling Algorithms In Cloud Computing} (A) Efficient Multi Queue Job Scheduling (MQS)

In Multi Queue Job Scheduling (MQS); based upon the burst time the scheduler groups the various client's jobs in the ascending order. They are processed and are placed in the different queues [4]. The Architecture of MQS is shown below:

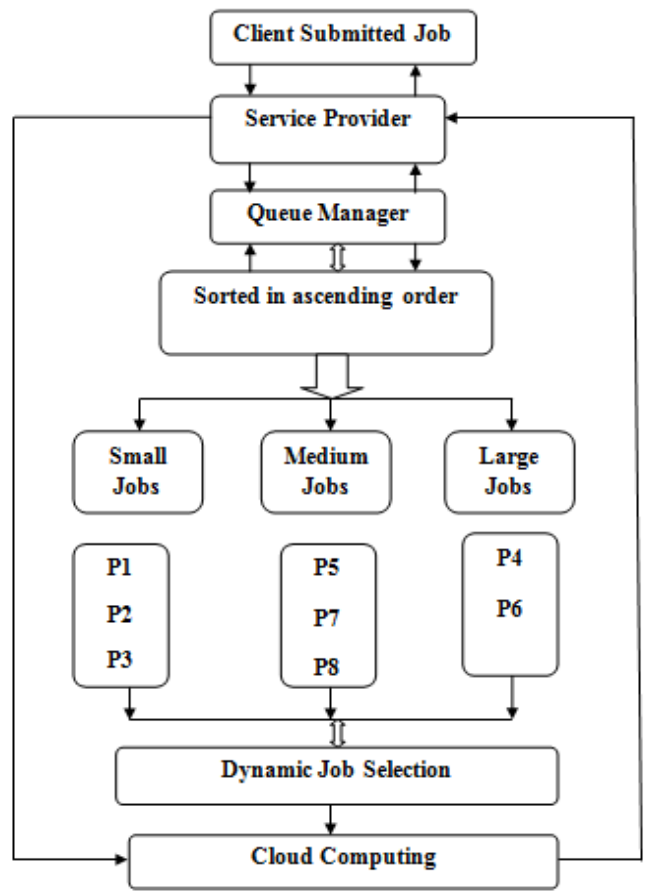

Figure 1: Architecture of MQS for Cloud Computing

Queue manager is responsible for providing the resources in the cluster. It maintains the list of all the available system that are in busy in processing the jobs by proper load balancing as well maintains list of systems who are idle and are ready to process jobs. It also indicates the scheduler to schedule the output of various jobs which is collected back by the queue manager [7]. Three queues are formed as small, medium and long which is based on ascending order measured in terms of burst time of the submitted client job.

- Small Queue: First $40 \%$ jobs are placed in it.

- Medium Queue: Next $40 \%$ of jobs are stored in the Medium Queue.

- Long Queue: Remaining $20 \%$ jobs are stored in it.

The execution of the jobs is carried out in the dynamic manner. The optimal allocation decreases time and availability of space in a productive manner without compensating the quality of the system [7].

\section{(B) Ant Colony Optimization (ACO)}

Ant colony optimization (ACO) is a meta-heuristic algorithm inspired from models of cooperative food search in ants [13]. ACO is inspired by a colony of ants that work together to find the shortest path between their nest and food source. Every ant will deposit a chemical substance called pheromone on the ground after they move from the nest to food sources and vice versa. This pheromone is used by the ants for communication purpose among themselves which further guides them to the next movement. On the basis of intensity of pheromone deposit on several path the ants determines the shortest distance from their nest to the desired food. The shorter path selected with have high pheromone value while the other paths will posses low pheromone value [6]. When the option given to the ants between a short path and a long path to the destination, the ants consistently found the shortest path after a period of time. To prevent establishing a suboptimal path (when the solution is trapped into a local minimum), the pheromone also evaporates over time, thus reducing the chance for other ants to take the path. On the other hand, the pheromone levels on the shortest path remain high because in this case, the pheromone deposit speed is faster than its evaporation speed [15].

As ACO is based upon the random search and due its complex characteristics it is used by the many researchers to solve NP-hard problems such as travelling salesman problem, optimization problems, graph coloring, vehicle routing problem and scheduling problem. The positive feedback mechanism, inner parallelism is the strength of the ACO algorithm [5] [13].

The Computational Flowchart of ACO is depicted below in Figure 2: 


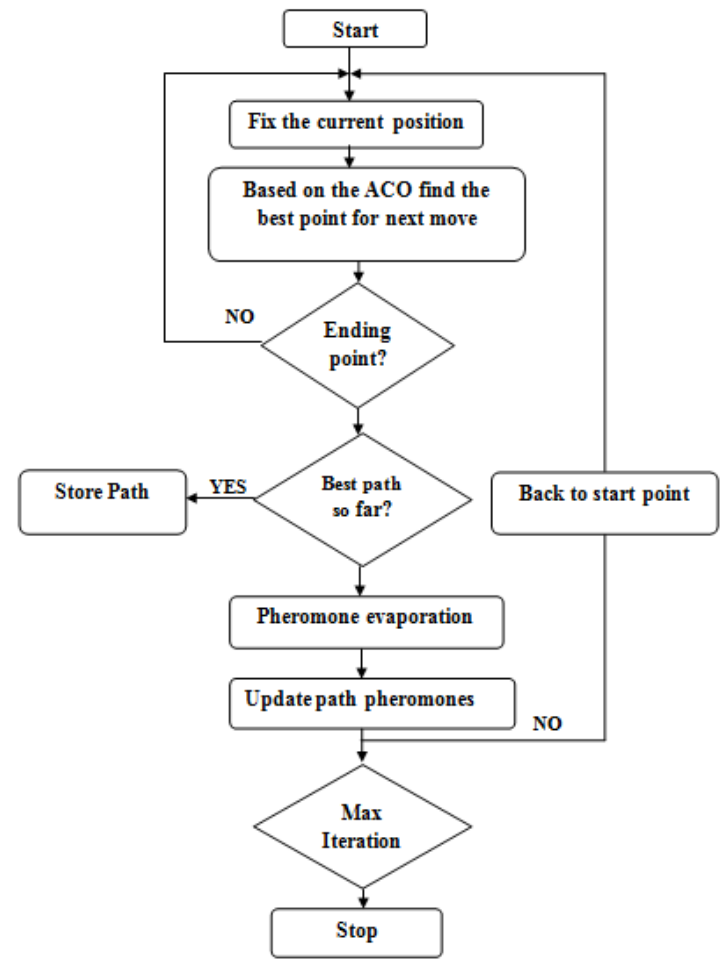

Figure 2: Computational Flowchart of ACO

\section{Experimental Results And Discussion}

We have implemented both heuristic base scheduling approaches Efficient MQS (Multi Level Queue Scheduling) Algorithm and ACO (Ant Colony Optimization) Algorithm in .Net Framework by establishing the cloud environment on fix number of virtual systems (which include system ram and processor) at an instance and by taking no of cloud user jobs to be scheduled as: 5, 10, 15 and 20. The energy consumption and time are parameters considered to compare the result of Efficient MQS with ACO.

Scenario I: When no of jobs to be scheduled is taken as 5; as Efficient MQS breaks the jobs in small, medium and long jobs while ACO is based upon the random search so we implemented both algorithms, the scheduler in each iteration schedule number of cloud user jobs in different order, the results obtained on best two iterations by both techniques are shown in Tabular form (Table 1) while figure 3(a) and 3(b) depict comparative analysis graph between two techniques. Clearly Efficient MQS Algorithm shows better performance in the terms of energy consumption and time consumed as compared with ACO Algorithm.

Table 1: Taking No of Jobs -5

Efficient MQS

$\mathrm{ACO}$

\begin{tabular}{|c|c|c|c|c|}
\hline No of Jobs & Energy & Time & Energy & Time \\
\hline \multirow{2}{*}{5} & 327 Joules & $26 \mathrm{~ms}$ & 605 Joules & $72 \mathrm{~ms}$ \\
\cline { 2 - 5 } & 351 Joules & $31 \mathrm{~ms}$ & 651 Joules & $72 \mathrm{~ms}$ \\
\hline
\end{tabular}

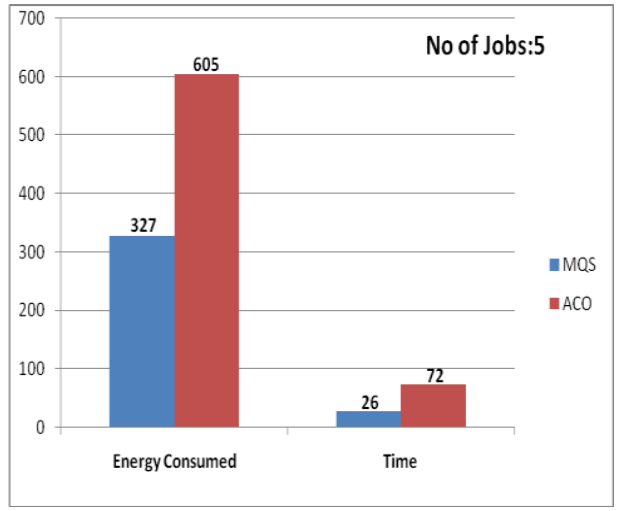

Figure: 3(a)

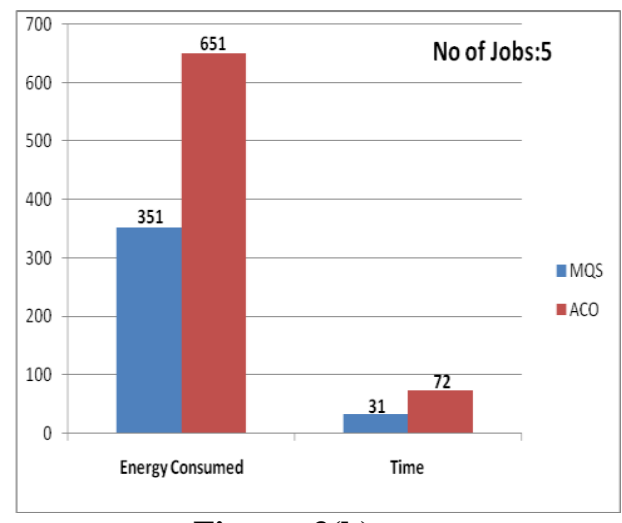

Figure: 3(b) 
Scenario II: When no of jobs to be scheduled is taken as 10; as MQS breaks the jobs in different jobs queue while ACO is based upon the random search, in each implementation rounds of both algorithms, the scheduler in each iteration arranges number of jobs in different sequence so the results obtained on best two iteration at an instance by both techniques are shown in Tabular form (Table 2) while figure 4(a) and 4(b) depict comparative analysis graph between two techniques. Clearly Efficient MQS Algorithm consumes less energy and time as compared with ACO Algorithm in each cycle.

Table 2: Taking No of Jobs - 10

Efficient MQS

\begin{tabular}{|c|l|c|c|c|}
\hline No of Jobs & Energy & Time & Energy & Time \\
\hline \multirow{2}{*}{$\mathbf{1 0}$} & 885 Joules & $58 \mathrm{~ms}$ & 2077 Joules & $146 \mathrm{~ms}$ \\
\cline { 2 - 5 } & 935 Joules & $59 \mathrm{~ms}$ & 2103 Joules & $146 \mathrm{~ms}$ \\
\hline
\end{tabular}

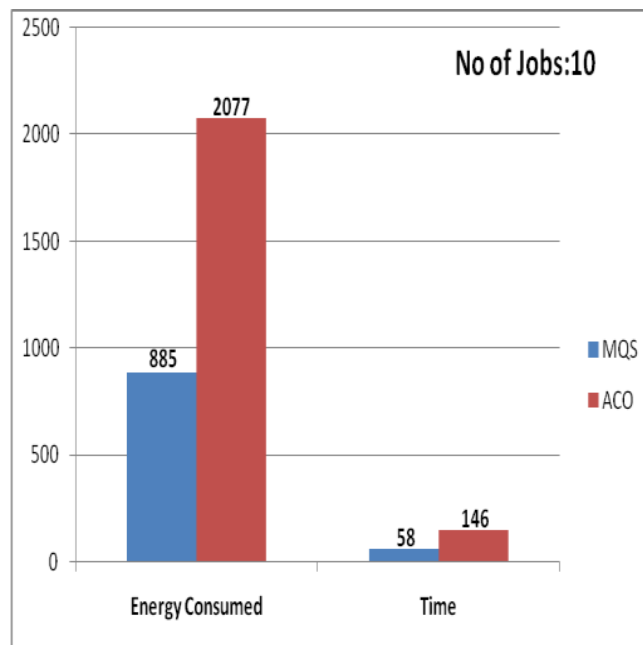

Figure: 4(a)

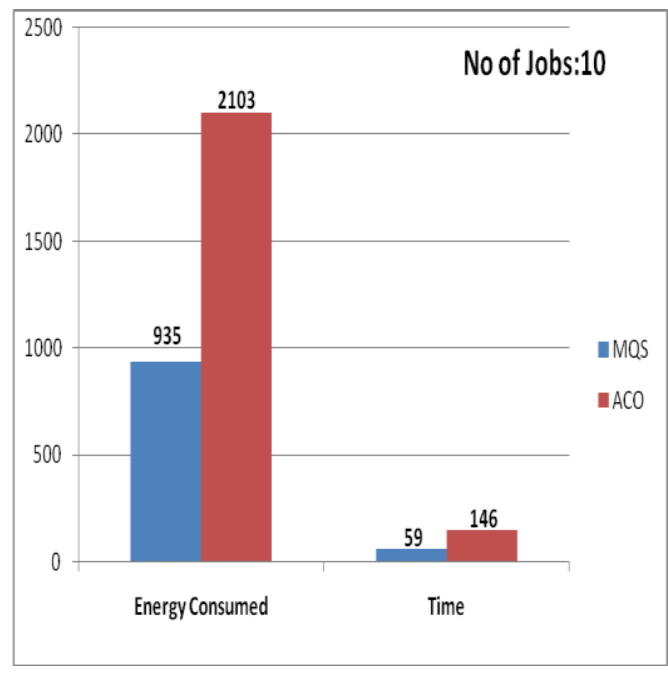

Figure: 4(b)

Scenario III: When no of cloud user jobs to be scheduled are taken as 15, the result obtained with best two iterations by both techniques is shown in Tabular form (Table 3) while figure 5(a) and 5(b) depict comparative analysis graph between two techniques. Clearly Efficient MQS Algorithm schedules the user jobs in better way when compared with ACO Algorithm in term of energy consumption and time.

Table 3: Taking No of Jobs - 15

Efficient MQS

$\mathrm{ACO}$

\begin{tabular}{|c|c|c|c|c|}
\hline No of Jobs & Energy & Time & Energy & Time \\
\hline \multirow{2}{*}{$\mathbf{1 5}$} & 1525 Joules & $68 \mathrm{~ms}$ & 3935 Joules & $211 \mathrm{~ms}$ \\
\cline { 2 - 5 } & 1548 Joules & $71 \mathrm{~ms}$ & 4010 Joules & $211 \mathrm{~ms}$ \\
\hline
\end{tabular}

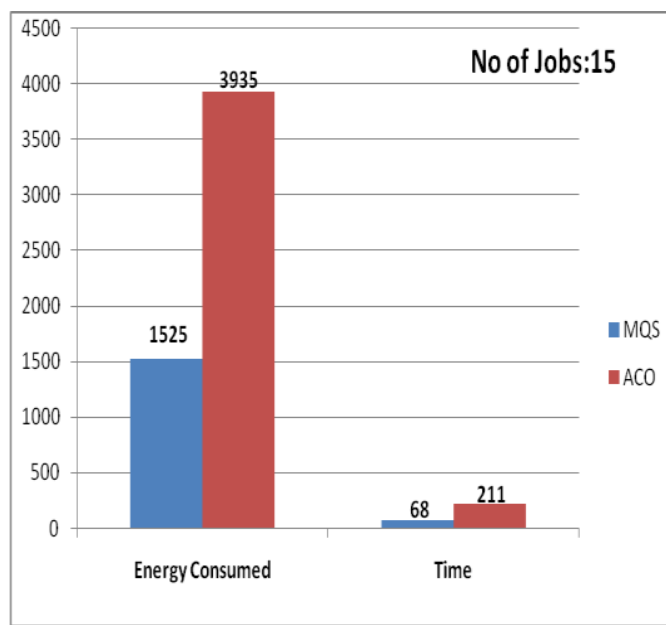

Fig: 5(a)

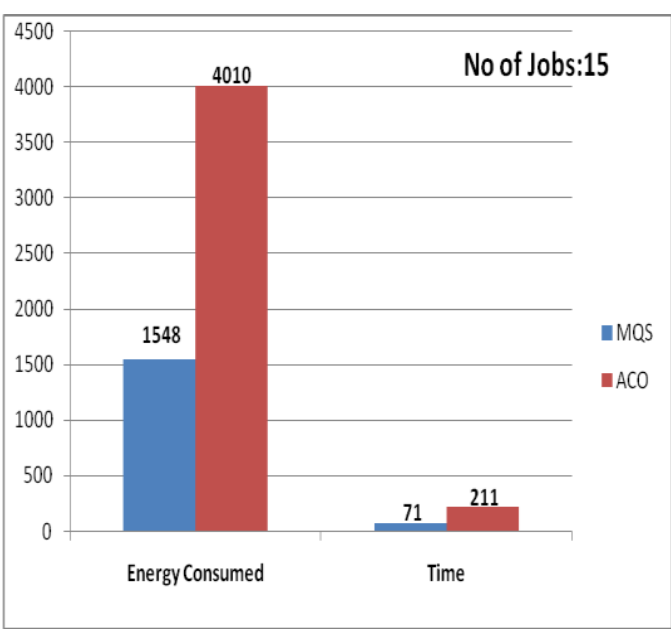

Fig: 5(b)

Scenario IV: When no of cloud user jobs to be scheduled are taken as 15 , the scheduler schedule number of jobs in different order in each execution, so the result obtained with two best iterations by both techniques is 
shown in Tabular form (Table 4) while figure 6(a) and 6(b) depict comparative analysis graph between two techniques. Clearly Efficient MQS Algorithm shows best results in the terms of energy consumption and limits execution time as compared with ACO Algorithm.

Table 4: Taking No of Jobs - 20

Efficient MQS

\begin{tabular}{|c|l|l|l|l|}
\hline No of Jobs & Energy & Time & Energy & Time \\
\hline \multirow{2}{*}{$\mathbf{2 0}$} & 2432 Joules & $95 \mathrm{~ms}$ & 6921 Joules & 281 ms \\
\cline { 2 - 5 } & 2527 Joules & $103 \mathrm{~ms}$ & 7124 Joules & 281 ms \\
\hline
\end{tabular}

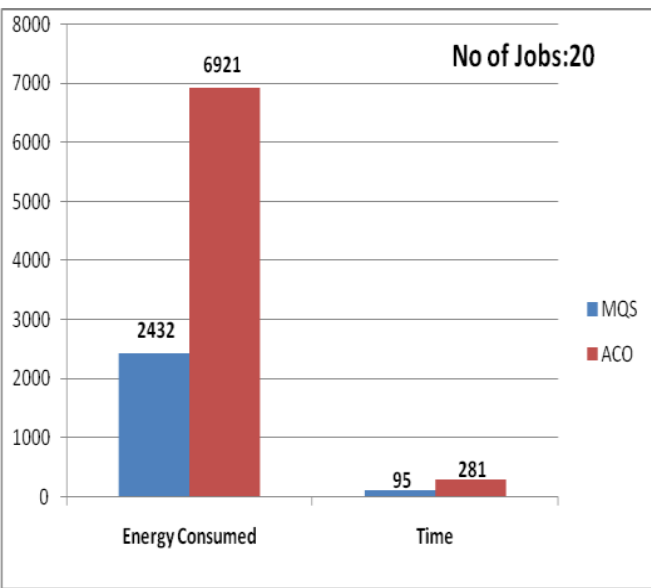

Fig: 6(a)

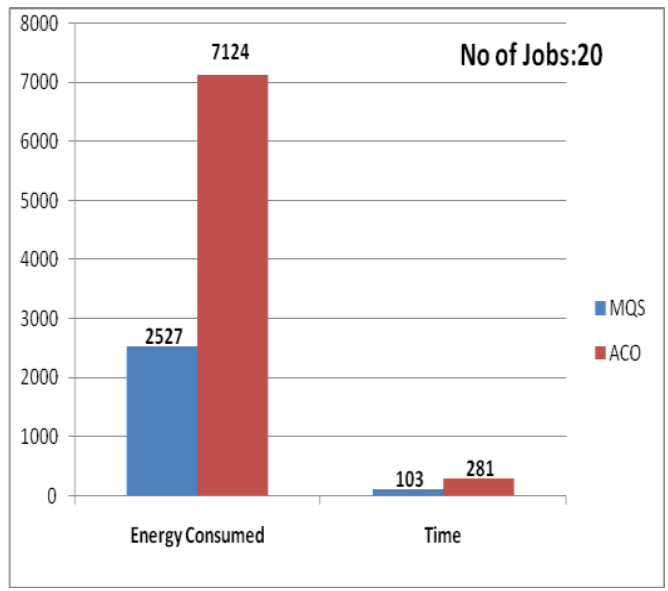

Fig: 6(b)

\section{Conclusion And Future Work}

In this paper the scheduling of cloud user jobs is done with the two heuristic based job scheduling algorithms Efficient MQS and ACO. The simulation result shows the superiority of Efficient MQS algorithm over ACO algorithm in terms of energy consumption and time over different number of cloud user jobs. The study done in this paper will be helpful for the researchers for selection of optimized job scheduling algorithm in cloud environment. In future the more work can be done in improving the framework of Efficient MQS so as to gain maximum profit in terms of energy consumption.

\section{References}

[1]. B.Kezia Rani, Dr.B.Padmaja Rani, Dr.A.Vinaya Babu "Cloud Computing and Inter-Clouds - Types, Topologies and Research Issues", Procedia Computer Science 50 (2015) 24 - 29, Elsevier, ISBCC 2015.

[2]. Deepak Puthal, B. P. S. Sahoo, Sambit Mishra, and Satyabrata Swain, "Cloud Computing Features, Issues and Challenges: A Big Picture", IEEE 2015

[3]. Abhishek Gupta, H S Bhadauriay, Annapurna Singhz and Jagdish Chandra Patni "A Theoretical Comparison of Job Scheduling Algorithms in Cloud Computing Environment", IEEE 2015.

[4]. V.Rajeshram, C.P. Shabariram, "Heuristics Based Multi Queue Job Scheduling for Cloud Computing Environment", International Journal of Research in Engineering and Technology, Volume 04, Issue 05, May 2015.

[5]. Medhat Tawfeek, Ashraf El-Sisi, Arabi Keshk and Fawzy Torkey, "Cloud Task Scheduling Based on Ant Colony Optimization", The International Arab Journal of Information Technology, Volume 12, No. 2, March 2015.

[6]. Rathnakar Achary, V. Vityanathan, Pethur Raj, and S. Nagarajan, "Dynamic Job Scheduling Using Ant Colony Optimization for Mobile Cloud Computing", Advances in Intelligent Systems and Computing, Springer International Publishing Switzerland 2015.

[7]. A V. Karthick, "An Efficient Multi Queue Job Scheduling for Cloud Computing”, IEEE world congress on computing and communication technology, 2014.

[8]. Yash P. Dave, Avani S. Shelat, Dhara S. Patel and Rutvij H. Jhaveri, "Various Job Scheduling Algorithms in Cloud Computing: A Survey", ICICES, IEEE 2014.

[9]. Teena Mathew, K. Chandra Sekaran, John Jose," Study and Analysis of Various Task Scheduling Algorithms in the Cloud Computing Environment", Page no: 658-664, IEEE 2014.

[10]. P Kowsik1, K.Rajakumari, "A Comparative Study on Various Scheduling Algorithms in Cloud Environment", International Journal of Innovative Research in Computer and Communication Engineering, November 2014.

[11]. Lokesh Kumar Arya, Amandeep Verma, "Workflow Scheduling Algorithms in Cloud Environment - A Survey", IEEE 2014.

[12]. Er. Shimpy, Mr. Jagandeep Sidhu, "Different Scheduling Algorithms in Different Cloud Environment", International Journal of Advanced Research in Computer and Communication Engineering, Vol. 3, Issue 9, September 2014.

[13]. Saurabh Bilgaiyan, Santwana Sagnika, Madhabananda Das, "An Analysis of Task Scheduling in Cloud Computing using Evolutionary and Swarm-based Algorithms", International Journal of Computer Applications (0975 - 8887), Volume 89 - No.2, March 2014

[14]. Pinal Salot, "A Survey of Various Scheduling Algorithm in Cloud Computing Environment", International Journal of Research in Engineering and Technology", Volume 02, Issue 02, Feb-2013.

[15]. Michael Brand, Michael Masuda, Nicole Wehner, Xiao-Hua Yu, "Ant Colony Optimization Algorithm for Robot Path Planning", IEEE 2010.

[16]. Dimitrios Zissis, Dimitrios Lekkas, “Addressing Cloud Computing Security Issues”, Future Generation Computer systems, Elsevier 2010. 